\title{
TEST OF THE OPTIMIZATION TECHNIQUES FOR THE PHOTOMETRIC ANALYSIS OF CONTACT BINARIES
}

\author{
E. LAPASSET, M. GOMEZ, and R. FARIÑAS \\ Observatorio Astronómico \\ Univ. Nacional de Córdoba \\ Laprida 854, 5000 Córdoba \\ Argentina
}

\begin{abstract}
We present light curve analyses of contact binaries and comparisons with previously published cross-correlations radial velocity data. The critical parameter $q$ (mass-ratio) obtained from a grid technique is confronted with the spectroscopic value. For total eclipsing systems, both values are always in a good or reasonable agreement, including some stars with shallow light curves. For partial eclipsing systems, convergent photometric and spectroscopic results are obtained for an important set of stars. Two exceptions are V523 Cas and XY Boo for which some arguments are suggested. We concluded that reliable parameter determinations can be obtained from pure photometric solutions, by means of grid techniques.
\end{abstract}

\section{Introduction}

The orbital, geometrical and physical parameters of contact binaries are determined almost exclusively by two different sets of observational data: photometric light curves and spectroscopic radial velocity curves. Both are necessary to completely describe the binary configuration. But, while differential photometry of eclipsing binaries is easily obtained, radial velocity measurements for this kind of stars present many difficulties, arising from the rotational width of the lines and the blending of both components. Only in the last years, by means of high dispersion spectrograms and cross-correlation techniques, those difficulties have been overcome. The question of the uniqueness of the solutions and the consistency of the results provided by the light and radial velocity curves are still controversial matters. The main and critical parameter relating both sets of data is the massratio $\left(q=m_{2} / m_{1}\right) ; q$ is directly determined by the amplitudes of the radial velocity curves of both components, and in general no objections are expressed about the uniqueness of the spectroscopic solutions. However, the limitations of radial velocity curves still arise from the difficulties in obtaining good, precise and dense diagrams. Concerning the photometric data, and in particular for partially eclipsing binaries the $q$ parameter is only indirectly determined and highly correlated with most of the other elements. The convergence of light curve solutions has been discussed and questioned many times, but photometric data continue to be the most expeditive and precise source of information especially for faint objects. So, the comparison between spectroscopic and photometric solutions and, particularly, between the two 
values of the mass-ratio continues to be of high interest. This is the main topic of the following sections.

\section{Light Curve Solutions}

Twenty years ago, synthetic light curve solutions revolutionized photometric analyses of eclipsing binary stars. In particular, the Wilson and Devinney's code (1971) based on the equipotential surface model was proven to be a strong tool for dealing with highly distorted contact systems. But when dealing with light curves sharing partial eclipses usually no convergent solutions are obtained. Thus some optimization techniques have to be employed to find a general minimum in the space of the adjustable parameters. One of these methods is the so-called "grid technique" which allows to select the best range of possible photometric values of $q$.

The aim of the present study is to evaluate the accuracy of detailed photometric solutions by comparing $q_{p h}$ and $q_{\text {op }}$. We found about twenty contact binaries with published radial velocity curves using modern cross-correlation techniques. But (to our surprise!) only a few of them had detailed analyses of their light curves. Consequently our first task was to perform grid photometric studies for some of them. The chosen stars were RR Cen, Y Sex, OO Aql, XY Boo, AB And and YY Eri. The two types of light curves were analyzed in different ways. For total eclipses, the grid analysis was not necessary because the geometric conditions, to produce occultations of a defined duration, force the convergence of the solutions (Lapasset and Sisteró 1984). In the case of partial eclipsing systems, it was unavoidable to apply some kind of optimization techniques. We employed the grid method and defined $S=\Sigma \omega r^{2}$ (sum of weighted squares of residuals) as the quality of solution parameter. In some cases the relation between $S$ and $q$ was clear and with a well-defined minimum (example: AB And, Fig. 1), but sometimes the $S$ vs. $q$ diagram was a flattened one making larger the mean error of $q$ and the correlated parameters (example: OO Aql, Fig. 2). Even though it was not obvious what the cause of these differences was, it is evident that the shallowness of the light curves renders difficult the obtaining of good photometric solutions.

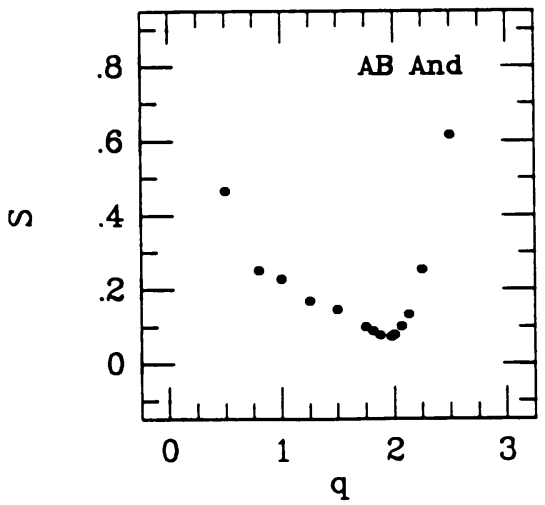

Figure 1. $S$ vs. $q$ for AB And.

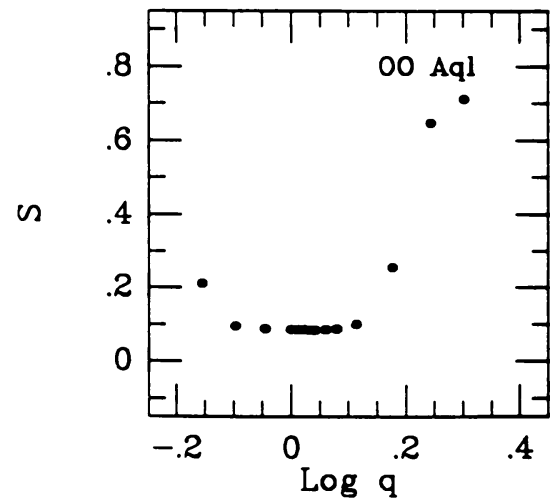

Figure 2. $S$ vs. $q$ for $\mathrm{OO}$ Aql.

Anyway, a mean photometric solution is always obtained and the range of reliable values of $q_{p h}$ has to be arbitrary determined in some way. We chose a range of $S$ 
within the $10 \%$ of its minimum value as acceptable, and this criterion was used to derive the mean error of $q_{p h}$.

\section{Results and Conclusions}

The results of our photometric analyses and those from other authors are listed in Table 1. This table shows successively the name and type of the stars, the mean depth of minima, and the mass-ratio values (photometric and spectroscopic). As it can be seen in the last columns for the total eclipsing systems, the coherence of $q_{p h}$ and $q_{s p}$ is quite satisfactory within the range of estimated (or published) errors. Small differences for isolated stars like AE Phe should be ascribed to unaccurate and scarce radial velocity data. Out of seven partial eclipsing binaries studied, five present similar values of $q$. The remaining two binaries are XY Boo and V523 Cas. XY Boo shows very shallow light curves and thus the photometric determination has to be considered highly uncertain. For V523 Cas $\left(q_{p h}=0.59\right.$ and $q_{s p}=0.42$ ) the following considerations should be taken in account: a) the analyzed light curve of V523 Cas is a single one, obtained without filters, while the Wilson and Devinney's code produces monochromatic synthetic light normals; b) both the photometric and spectroscopic solutions represent a W-type contact binary and the difference between the values of $q$ does not invalidate the photometric determination of the remaining parameters (inclination, degree of contact, etc.) which do not vary too much passing from $q=0.59$ to $q=0.42$; and finally, c) as result of our comparison between $q_{s p}$ and $q_{p h}$ for contact binaries with total eclipses and as consequence of many light curve analyses published up till now for several types of binaries, it must be concluded that an astrometric determination of $q$ is not possible. However with regard to the evolutionary status of W UMa stars some very important characteristics such as the $A$ or $W$ type, the estimated value of the mass-ratio, and the degree of contact are of high interest. And these parameters can be obtained from the photometric data even when the results are not very precise, as for instance, in the case of V523 Cas.

Table 1. Photometric and spectroscopic mass-ratio values for the studied sample of W Umas.

\begin{tabular}{|c|c|c|c|c|c|c|c|}
\hline Star & Ecl. & $\mathbf{I y p}_{\mathbf{y}}$ & $\Delta=$ & $q_{1 p}$ & Lef. & $q_{p h}$ & Lef. \\
\hline $75660 \mathrm{ph}$ & tot. & $\Lambda$ & 0.45 & $0.24 \pm 0.03$ & (1) & $0.237 \pm 0.001$ & (5) \\
\hline ID URa & $"$ & $\Lambda$ & 0.23 & $0.07 \pm 0.02$ & (2) & $0.071 \pm 0.001$ & (12) \\
\hline In Cen & $"$ & $\Lambda$ & 0.11 & $0.21 \pm 0.01$ & (3) & $0.17 \pm 0.02$ & (13) \\
\hline I Sex & $"$ & $\Lambda$ & 0.92 & $0.18 \pm 0.03$ & (1) & $0.18 \pm 0.01$ & (13) \\
\hline cC Con & $"$ & $\mathbf{\nabla}$ & 0.80 & $0.47 \pm 0.04$ & (4) & $0.62 \pm 0.01$ & (14) \\
\hline DVIa & $"$ & $\mathbf{\nabla}$ & 0.65 & $0.49 \pm 0.03$ & (2) & $0.45 \pm ?$ & (15) \\
\hline L2 Con & $"$ & $\mathbf{\nabla}$ & 0.70 & $0.43 \pm 0.03$ & (4) & $0.42 \pm 0.01$ & (12) \\
\hline IE Phe & $"$ & $\nabla$ & 0.65 & $0.47 \pm 0.01$ & (5) & $0.39 \pm 0.01$ & (5) \\
\hline $00 \triangle q 1$ & parc. & $\Lambda$ & 0.92 & $0.84 \pm 0.01$ & (6) & $1.04 \pm 0.22$ & (13) \\
\hline IY Boo & $"$ & $\Lambda$ & 0.33 & $0.16 \pm 0.04$ & (4) & $1.00 \pm ?$ & (13) \\
\hline AB And & $"$ & $\nabla$ & 0.75 & $0.49 \pm 0.01$ & (7) & $0.51 \pm 0.02$ & (13) \\
\hline II Eri & $"$ & $\nabla$ & 0.68 & $0.40 \pm 0.05$ & (8) & $0.44 \pm 0.03$ & (13) \\
\hline V523 Cas & $"$ & $\mathbf{\nabla}$ & 0.80 & $0.42 \pm 0.03$ & (9) & $0.59 \pm 0.06$ & (16) \\
\hline J Pog & $"$ & $\nabla$ & 0.54 & $0.33 \pm 0.01$ & (10) & $0.33 \pm 0.03$ & (17) \\
\hline BI Dra & $"$ & $\nabla$ & 0.45 & $0.28 \pm 0.01$ & (11) & $0.33 \pm 0.05$ & (18) \\
\hline
\end{tabular}

References:

(1)McLean(1983); (2)McLean(1981); (3)King \& Hilditch(1984); (4)McLean \& Hilditch(1983); (5)Van Hamme \& Wilson(1985); (6)Hrivnak(1989); (7)Hrivnak(1988); (8)Nesci et al.(1986); (9)Milone et al.(1985); (10)Lu(1985); (11)Batten \& Lu(1986); (12)Wilson \& Devinney(1973); (13)this paper; (14)Rucinski(1976); (15)Hilditch(1981); (16)Maceroni(1986); (17)Zhai et al.(1984); (18)Rucinski \& Kaluzny(1982). 
The final remark concerning the presented confrontation between photometric and spectroscopic data through the derived values of $q$ (as summarized in Fig. 3 ) is quite conclusive; both solutions are in good agreement, within the range of errors, in most of the cases; some precautions ought to be taken concerning the light curves with partial eclipses, particularly if they are too shallow. But from the present study of a selected sample of contact binaries it is concluded that reliable results can be obtained from pure photometric solutions by means of grid techniques.

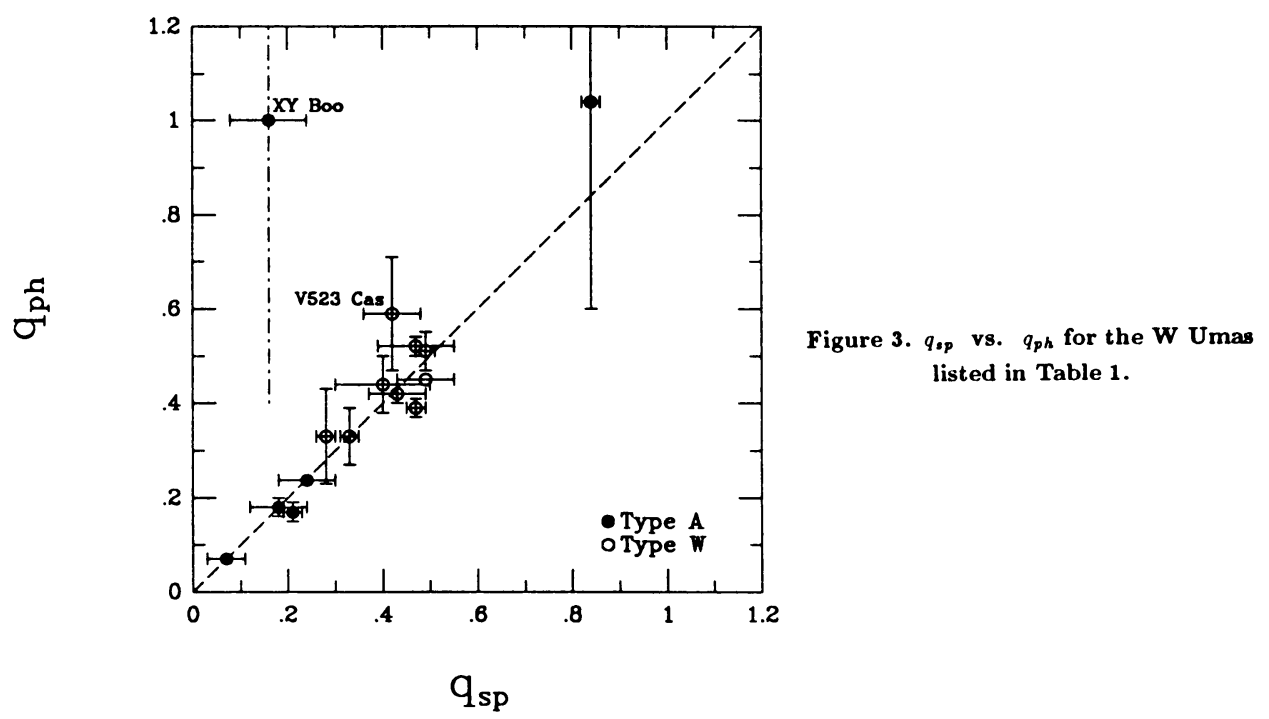

\section{References}

Batten, A.H., and Lu, W. 1986. Publ. Astron. Soc. Pacific 98, 92.

Hilditch, R.W. 1981. Mon. Not. Roy. Astron. Soc. 196, 305.

Hrivnak, B.J. 1988. Astrophys. J. 335, 319.

Hrivnak, B.J. 1989. Astrophys. J. 340, 458.

King, D.J., and Hilditch, R.W. 1984. Mon. Not. Roy. Astron. Soc. 209, 645.

Lapasset, E., and Sisteró R.F. 1984. Astron. Astrophys. 130, 97.

Lu, W. 1985. Publ. Astron. Soc. Pacific 97, 1086.

Maceroni, C. 1986. Astron. Astrophys. 170, 43.

McLean, B.J. 1981. Mon. Not. Roy. Astron. Soc. 195, 931.

McLean, B.J. 1983. Mon. Not. Roy. Astron. Soc. 204, 817.

McLean, B.J., and Hilditch, R.W. 1984. Mon. Not. Roy. Astron. Soc. 209, 645.

Milone, E.F., Hrivnak, B.J., and Fischer, W.A. 1985. Astron. J. 90, 354.

Nesci, R., Maceroni, C., Milone, L., and Russo, G. 1986. Astron. Astrophys. $159,142$.

Rucinski, S.M. 1976. Publ. Astron. Soc. Pacific 88, 777.

Rucinski, S.M., and Kaluzny, J. 1982. Astrophys. Space Sci. 88, 433.

Van Hamme, W., and Wilson, R.F. 1985. Astron. Astrophys. 152, 25.

Wilson, R.E., and Devinney, E.J. 1971. Astron. Astrophys. 166, 605.

Wilson, R.E., and Devinney, E.J. 1973. Astron. Astrophys. 182, 539.

Zhai, D., Leung, K-C., and Zhang, R. 1984. Astron. Astrophys. Suppl. Ser. $\mathbf{5 7}, 487$. 